\title{
NHŨ̉NG THUẬN LỘI VÀ KHÓ KHĂN TRONG VIỆC TÍCH HỢP ĐỀ CƯƠNG THEO HƯỚNG CDIO VÀ AUN VÀO VIỆC GIẢNG DẠY CỦA GIẢNG VIÊN TRƯỜnG ĐẠI HỌC THỦ DẦ MỘT Nguyễn Thị Mai ${ }^{(*)}$
}

(*) Thạc sĩ. Truờng Đại học Thủ Dầu Một. Email: maint@tdmu.edu.vn.

DOI: $10.37550 /$ tdmu.CFR/2021.01.130

\section{Tóm tắt}

Trong bối cảnh toàn cầu hóa, hội nhập kinh tế quốc tế diễn ra mạnh mẽ, hơn bao giờ hết ngành giáo dục đứng trước nhũ̃ng thuận lợi và thách thức vô cùng to lớn. Thuận lợi đó chính là sụ hỗ trợ tù công nghệ, nguồn học liệu đa dạng. Tuy nhiên khó khăn là ngành giáo dục phải làm sao để tạo ra nguồn nhân lực có chất luợng để đáp úng nhu cầu của xã hội. Được thành lập tù̀ năm 2009 đến nay Trương Đại học Thủ Dầu Một cũng không tránh được nhũng khó khăn chung đó và nhà trường đã có nhũng thay đổi không ngùng để bắt kịp tình hình mới. Trong bối cảnh đó bài viết phân tích nhũng thuận lợi và khó khăn của giảng viên đại học Thủ Dầu Một trong việc tích hơp đề cuơng theo hướng CDIO và AUN nhu là một trong nhũng giải phải pháp để nâng cao chất lương giảng dạy của Trương Đại học Thủ Dầu Một.

Từ khóa: Tích hợp đề cuoong, giảng dạy theo CDIO và $A U N$

\section{1. Đặt vấn đề}

Trong những năm gần đây khi nền kinh tế Việt Nam đã thu được nhiều thành tựu đáng kể, chất lượng cuộc sống người dân không ngừng được nâng cao. Vai trò của các trường đại học như những động lực cho sự tăng trưởng kinh tế càng trở nên quan trọng hơn bao giờ hết. Tuy nhiên, nhiều khảo sát và đánh giá chất lượng giáo dục đại học của Bộ GD\&ĐT đã cho thấy sự thay đổi của giáo dục đại học chưa theo kịp nhu cầu của kinh tế xã hội. Do đó, thực trạng này đã thúc đẩy nhiều nỗ lực để tiếp tục nâng cao chất lượng hệ thống giáo dục đại học. Đứng trước những thời cơ và thách thức của nền giáo dục nước nhà, Trường Đại học Thủ Dầu Một cũng không ngừng thay đổi, luôn tiếp cận theo hướng mới để nâng cao chất lượng đào tạo, đáp ứng được nhu cầu lao động của xã hội. Minh chứng cụ thể nhất cho vấn đề là nhà trường đã triển khai cả hướng tiếp cận đề cương theo hướng CDIO và AUN. Liệu hai cách tiếp cận này có mâu thuẫn và gây khó khăn cho giảng viên trong quá trình giảng dạy? Bài viết này sẽ phân tích những thuận lợi và khó khăn của giảng viên Đại học Thủ Dầu Một trong việc tiếp cận đề cương theo hướng $\mathrm{CDIO}$ và $\mathrm{AUN}$.

\section{Nhận thức về CDIO và $\mathrm{AUN}$}




\subsection{CDIO và lợi ích của CDIO}

CDIO là viết tắt của cụm từ tiếng Anh Conceive - Design - Implement - Operate, nghĩa là: Hình thành ý tưởng, thiết kế ý tưởng, thực hiện và vận hành. Và CDIO là một đề xướng của các khối ngành kỹ thuật thuộc ĐH Kỹ thuật Massachusetts, Mỹ, phối hợp với các trường đại học Thụy Điển ${ }^{[1]}$. Đây là một giải pháp nâng cao chất lượng đào tạo đáp ứng yêu cầu xã hội trên cơ sở xác định chuẩn đầu ra để thiết kế chương trình và phương pháp đào tạo theo một quy trình khoa học. Bên cạnh đó, CDIO được xây dựng một cách hợp lý, logic và về phương pháp tổng thể mang tính chung hóa có thể áp dụng để xây dựng quy trình chuẩn cho nhiều lĩnh vực đào tạo khác nhau.

Chính vì vậy nếu áp dụng theo chương trình CDIO sẽ có những lợi ích:

Thứ nhất, Đào tạo theo cách tiếp cận $\mathrm{CDIO}$ gắn với nhu cầu của người nhà tuyển dụng, từ đó giúp thu hẹp khoảng cách giữa đào tạo của nhà trường và yêu cầu của các nhà sử dụng nguồn nhân lực;

Thứ hai, Đào tạo theo cách tiếp cận CDIO giúp người học phát triển toàn diện với các "kỹ năng cứng" và "kỹ năng mềm" để nhanh chóng thích ứng với môi trường làm việc luôn thay đổi;

Thứ ba, Đào tạo theo cách tiếp cận CDIO sẽ giúp các chương trình đào tạo được xây dựng và thiết kế theo một quy trình chuẩn. Các công đoạn của quá trình đào tạo sẽ có tính liên thông và gắn kết chặt chẽ;

Thứ tư, Cách tiếp cận CDIO là cách tiếp cận phát triển, gắn phát triển chương trình với chuyển tải và đánh giá hiệu quả giáo dục đại học, góp phần nâng cao chất lượng giáo dục đại học lên một tầm cao mới.

\subsection{AUN và lọi ích của $A U N$}

AUN là đánh giá chất lượng giáo dục đại học theo những tiêu chuẩn đảm bảo chất lượng chung của khu vực ASEAN (ASEAN University Network - Quality Assurance, viết tắt là AUN-QA). Đây cũng là cách mà mạng lưới các trường đại học ASEAN nâng cao sự tin tưởng lẫn nhau về chất lượng đào tạo giữa các trường trong khu vực cũng như với các trường đại học đối tác trên thế giới, từng bước góp phần thúc đẩy sự công nhận thành quả học tập và phát triển hợp tác giữa các trường đại học trong khu vực Đông Nam Á. Việc lựa chọn kiểm định theo chuẩn $\mathrm{AUN}$ nhằm giúp các trường biết chương trình đào tạo đã đạt đến cấp độ nào trên thang đánh giá của khu vực. Tiếp nữa, phát hiện chương trình còn tồn tại những gì cần khắc phục nhằm đảm bảo chương trình đạt chất lượng ngang tầm các chương trình cùng lĩnh vực trong khu vực ASEAN.

Bộ tiêu chuẩn của AUN-QA có 18 tiêu chuẩn với 74 tiêu chí. Mỗi tiêu chí được đánh giá theo 7 mức là: 1 "không có gì (không có tài liệu, kế hoạch, minh chứng )"; 2 "chủ đề này của hệ thống đảm bảo chất lượng bên trong mới chỉ nằm trong kế hoạch"; 3 "có tài liệu, nhưng không có minh chứng rõ ràng"; 4 "có tài liệu và minh chứng"; 5 "có minh chứng rõ ràng về hiệu quả trong lĩnh vực xem xét"; 6 "chất lượng tốt"; 7 "xuất sắc" [1]. Mỗi tiêu chí trong bộ tiêu chuẩn đều có trọng số như nhau, điểm đánh giá của toàn bộ 
chương trình là điểm trung bình cộng của cả 74 tiêu chí. 4.0 là ngưỡng điểm tối thiểu để đạt tiêu chuẩn kiểm định chất lượng của AUN.

Việc hướng tới và đạt được tiêu chuẩn AUN không chỉ đem lại lợi ích cho nhà trường, sinh viên mà cả người sử dụng lao động. Đối với nhà trường, thông qua hoạt động tự đánh giá và đánh giá ngoài, những điểm mạnh và điểm tồn tại của chương trình đào tạo đã được chỉ ra, và nhà trường phải có kế hoạch hành động cụ thể để cải tiến khắc phục những tồn tại này. Đây là cái được lớn nhất từ phía nhà trường. Đồng thờ sinh viên và giảng viên được hưởng lợi vì môi trường dạy và học được cải thiện, nâng cấp và đảm bảo chất lượng. Ngoài ra, nhờ kiểm định chất lượng theo chuẩn AUN, nhà trường xác định được vị thế của chương trình đào tạo trong khu vực và làm cơ sở để xây dựng lộ trình phát triển cho chương trình đào tạo theo hướng vươn tới các chuẩn mực quốc tế.

Bên cạnh đó, khi áp dụng chuẩn $\mathrm{AUN}$ bộ tiêu chuẩn này không tập trung vào những đặc trưng riêng của từng chuyên ngành mà tập trung đánh giá những điều kiện để đảm bảo chất lượng đào tạo của một chương trình. Bộ tiêu chuẩn tập trung và những lĩnh vực mà bất kỳ chương trình đạo tạo bậc đại học nào cũng có như chuẩn đầu ra; khung chương trình; giảng viên và sinh viên, cơ sở vật chất, công tác đảm bảo chất lượng...

Trong khi đó, sinh viên là đối tượng được hưởng lợi rất lớn từ hoạt động đánh giá này, bởi được học tập trong một môi trường được cải tiến liên tục và đảm bảo chất lượng. Kết quả kiểm định $\mathrm{AUN}$ như một sự khẳng định chương trình đào tạo với xã hội về chất lượng sản phẩm đầu ra của chương trình, vì thế chắc chắn sinh viên tiếp cận việc làm phù hợp dễ dàng hơn. Trong tương lai gần, tham gia kiểm định chương trình đào tạo theo chuẩn AUN-QA còn tạo điều kiện cho sinh viên được chuyển đổi tín chỉ học tập giữa các chương trình đào tạo của các trường đại học thành viên $\mathrm{AUN}$, tạo lợi thế cho hoạt động trao đổi sinh viên, giảng viên giữa các trường đại học. Và cuối cùng, người sử dụng lao động có một cơ sở tin cậy để tìm kiếm nguồn nhân lực đảm bảo chất lượng, từ một chương trình đào tạo có những tham chiếu chất lượng mang tính quốc tế.

\section{Những thuận lợi và khó khăn đối với giảng viên Trường Đại học Thủ Dầu Một khi xây dụng đề cương môn học theo hướng tích hợp chuẩn CDIO và $A U N$}

\subsection{Thuận lọi khi áp dụng xây dụng chuẩn đầu ra theo hướng tích hợp CDIO và AUN của giảng viên Trường ĐH Thủ Dầu Một}

Điểm thuận lợi đầu tiên trong vấn đề tích hợp đề cương theo hướng CDIO và AUN có thể nói là thuận lợi về mặt tư tưởng - một điều rất khó khi nhà trường bắt đầu áp dụng theo hướng CDIO. Khi trường Đại học Thủ Dầu Một tiến hành thực hiện viết đề cương theo hướng $\mathrm{CDIO}$ giảng viên chưa quen với cách làm mới nên giảng viên ái ngại với việc thay đổi. Nhưng khi đã thành thạo đề cương theo hướng CDIO giảng viên nhận thấy đề cương theo hướng này có chuẩn đầu ra rất rõ ràng, xác lập được mục tiêu giảng dạy cụ thể không còn mang tính mơ hồ, chung chung như trước đây. Vì vậy khi tiếp cận đề cương theo hướng AUN giảng viên không ngại việc thay đổi, sẵn sàng thay đổi đây là thuận lợi đầu 
tiên khi nhà trường vận dụng đề cương theo hướng AUN nhưng tích hợp cả CDIO.

Thuận lợi thứ hai đó là đề cương theo hướng $\mathrm{CDIO}$ và $\mathrm{AUN}$ hoàn toàn không trái ngược và có thể nói là bổ trợ nhau. Nếu như đề cương theo hướng CDIO xác định chuẩn đầu ra cụ thể rõ ràng thì đề cương theo hướng $\mathrm{AUN}$ còn xác định được chuẩn đầu ra cho toàn bộ chương trình đào tạo và các phần nhỏ phải thống nhất trong một hệ thống. Hơn thế nữa, đề cương theo hướng $\mathrm{AUN}$ có thể áp dụng cho các khối ngành giúp khắc phục hạn chế của CDIO là thiên về khối ngành kỹ thuật. Tuy nhiên, chính đề cương theo hướng CDIO là nền tảng để giảng viên đại học Thủ Dầu Một có cơ sở để tiếp cận đề cương theo hướng AUN và tích hợp cả hai đề cương trong quá trình giảng dạy.

Thuận lợi thứ ba, đó là lãnh đạo nhà trường luôn khuyến khích động viên giảng viên trong quá trình tiếp cận phương pháp mới, thành lập những tổ chuyên môn, tổ chức những những khóa tập huấn để hỗ trợ giảng viên, nếu giảng viên có vấn đề khó khăn đội ngũ chuyên môn của nhà trường luôn sẵn sàng hỗ trợ.

Thuận lợi thứ tư, những tài liệu về $\mathrm{CDIO}$ cũng như AUN được nhà trường thường xuyên cập trên website của trường để giảng viên dễ dàng tiếp cận tài liệu.

Thuận lợi cuối cùng để đảm bảo cho việc giảng dạy cho phương pháp theo hướng tiếp cận CDIO đến AUN yêu cầu cần có sự thay đổi lớn về cơ sở vật chất, trang thiết bị phòng học nên nhà trường đã không ngừng cải thiện cơ sở vật chất, môi trường học tập để đáp ứng cho giảng viên và sinh điều kiện giảng dạy, học tập tốt nhất.

\subsection{Nhũng khó khăn khi áp dụng xây dụng chuẩn đầu ra theo hướng tích hợp CDIO và AUN của giảng viên Truò̀ng $\boxplus H$ Thủ Dầu Một}

Khó khăn đầu tiên của việc tích hợp đề cương theo hướng $\mathrm{CDIO}$ và $\mathrm{AUN}$ là giảng viên phải làm việc nhiều hơn trong quá trình soạn đề cương và việc giảng dạy. Đối với đề cương theo hướng CDIO phải trải qua 4 bước: hình thành ý tưởng, thiết kế, triển khai và vận hành, 4 bước này khối ngành Khoa học xã hội rất khó để áp dụng. Tiếp theo đối với đề cương theo hướng AUN giảng viên phải xác định từng chuẩn đầu ra của từng học phần nhỏ và phải đặt chuẩn đầu ra đó trong tổng thể chương trình đào tạo để trách sự trùng lắp. Trong khi đó có những giảng viên một số ngành do số lượng sinh viên đông, giảng viên phải thường xuyên lên lớp nên việc bổ sung, cập nhật đảm bảo đề cương cũng là một vấn đề khó khăn.

Một khó khăn nữa của giảng viên đại học Thủ Dầu Một là mặc dù nhà trường đã có nhiều bổ sung cập nhật về nguồn tài liệu, cơ sở vật chất nhưng đôi khi điều kiện về nguồn học liệu, thiết bị máy móc, phòng thí nghiệm vẫn chưa đáp ứng được nhu cầu giảng dạy và học tập. Bên cạnh đó, với đề cương theo chuẩn AUN là mức đánh giá của khu vực trong khi đó sinh viên của trường Đại học Thủ Dầu Một mặt bằng chung của sinh viên còn khá thấp so với sinh viên của một số thành phố lớn như TP Hồ Chí Minh, Hà Nội nên cũng có nhiều khó khăn cho giảng viên khi áp dụng các chuẩn của khu vực.

Hơn thế nữa đội ngũ giảng viên của Trường Đại học Thủ Dầu Một đến từ nhiều nơi khác nhau trên cả nước nên trong quá trình công tác vì điều kiện cá nhân một số giảng viên không gắn kết lâu dài với nhà trường, đều này cũng dẫn đến quá trình thực hiện những mục tiêu lâu dài của nhà trường bị ảnh hưởng. 


\section{Kết luận và kiến nghị}

Rõ ràng việc vận dụng đề cương theo hướng tiếp cận theo hướng CDIO và AUN là một quyết định đúng đắn của nhà trường, góp phần giải đáp những vấn đề của công tác đào tạo nguồn nhân lực bối cảnh mới. Và việc áp dụng đề cương theo hướng CDIO và AUN không những không mâu thuẫn nhau mà còn hỗ trợ nhau cả về kiến thức, kỹ năng, thái độ và môi trường đào tạo.

Tuy nhiên để việc vận dụng tích hợp đề cương theo hướng CDIO và AUN thiết nghĩ trong thời gian tới giảng viên và nhà trường cần có những cải tiến sau:

Về phía nhà trường: cần thường xuyên có những khóa tập huấn, trao đổi kinh nghiệm giữa giảng viên và đội ngũ chuyên gia để chia sẽ, trao đổi kinh nghiệm cũng như giúp đỡ giảng viên tháo gỡ những vướng mắc; cải thiện thêm hệ thống cơ sở vật chất đặc biệt là hệ thống phòng thí nghiệm, máy móc, trang thiết bị, hệ thống wifi; nên có những buổi nói chuyện chuyên đề, định hướng nghề để nâng cao ý thức học tập của sinh viên để sinh viên ý thức được tầm quan trọng của việc học, hiểu được những nhu cầu của thị trường, của nhà tuyển dụng để bổ sung những kiến thức, kỹ năng cần có để tăng cơ hội có việc làm sau khi tốt nghiệp ra trường.

Về phía giảng viên: Trước hết giảng viên phải có tinh thần cầu thị, không ngại khó trong việc cập nhật kiến thức, phương pháp để nâng cao chất lượng giảng dạy.

Bên cạnh đó, giảng viên cũng nên xâm nhập thực tế, hiểu nhu cầu xã hội để giúp nhà trường có hướng tiếp cận trong giảng dạy phù hợp với tình hình thực tế để tạo ra nguồn nhân lục đáp ứng được nhu cầu thực tế,

Cuối cùng là người làm việc trực tiếp với sinh viên, giảng viên phải hiểu tâm tư nguyện vọng của các em để giúp các em hứng thú với việc học tập cũng như phát hiện ta những tiềm năng của các em để các em khó thể phát huy hết những điểm mạnh của mình.

\section{Tài liệu tham khảo}

[1] AUN-QA: Cơ sở đáng tin cậy cho việc chọn lựa trường của phụ huynh và học sinh, https://tuoitre.vn/aunqa-co-so-dang-tin-cay-cho-viec-chon-luatruong-cua-phu-huynh-va-hoc-sinh-1140328.htm, truy cập ngày 27/11/2020

[2] Lê Hoài Bắc, Hồ Bảo Quốc, Một số kinh nghiệm xây dựng đề cuoong theo chuẩn CDIO, http://www.vnuhcm.edu.vn

[3] Đề xướng bộ tiêu chuẩn CDIO, www.tdmu.edu.vn

[4] Đại học Quốc gia thành phố Hồ Chí Minh (2012), Đào tạo đáp úng nhu cầu xã hội và hội nhập quốc tế, Hội nghị CDIO toàn quốc.

[5] Đại học quốc gia Hồ Chí Minh(2006), Tài liệu đánh giá cấp chương trình theo chuẩn AUN, NXB ĐH QG HCM 
[6] Chuẩn ra chương trình đào tạo www.tdmu.edu.vn

[7] Khoa Cơ khí, Đại học Quốc gia thành phố Hồ Chí Minh, Hướng dẫn đối sánh chương trình đào tạo hiện hành theo 12 tiêu chuẩn $\mathrm{CDIO}$.

[8] Hồ Tấn Nhựt, Đoàn Thị Minh Trinh (Biên dịch 2009), Cải cách và xây dựng chuơng trình đào tạo kỹ thuật theo phwơng pháp tiếp cận CDIO, Nxb Đại học Quốc gia TP.HCM.

[9] Phương pháp viết đề cương môn học theo hướng CDIO, www.tdmu.edu.vn

[10] Tổng quan về chương trình đào tạo, www.tdmu.edu.vn

[11]Tổng quan về CDIO, https://tdmu.edu.vn/cdio/tong-quan-cdio-1/tongquan-ve-cdio, truy cập ngày 09 tháng 12 năm 2020.

[12]Đoàn Thị Minh Trinh, Nguyễn Hội Nghĩa, 2013, Hương dẫn thiết kế và phát triển chưong trình đào tạo đáp úng chuẩn đầu ra, Nxb Đại học Quốc gia TP.HCM. 